Proceedings of the National Conference on Neutron Scattering and the Complementary Methods in the Investigations of the Condensed Phases, Chlewiska 2007

\title{
Properties of Neutron Doped Multicrystalline Silicon for Solar Cells
}

\author{
C. Pochrybniak ${ }^{a, *}$, K. Pytel ${ }^{a}$, J.J. MilczareK ${ }^{a}$, \\ J. JARoszeWicZ ${ }^{a}$, M. LiPiński ${ }^{b}$, T. Piotrowski ${ }^{c}$ \\ AND J. KANSY ${ }^{d}$ \\ ${ }^{a}$ Institute of Atomic Energy, Świerk, 05-400 Otwock, Poland \\ ${ }^{b}$ Institute of Metallurgy and Materials Science \\ Polish Academy of Sciences \\ Reymonta 25, 30-059 Cracow, Poland \\ ${ }^{c}$ Institute of Electron Technology, Warsaw, Poland \\ ${ }^{d}$ University of Silesia, Bankowa 12, 40-007 Katowice, Poland
}

Dedicated to Professor Jerzy Janik on the occasion of his 80th birthday

The technology of neutron transmutation doping of silicon wafers in MARIA nuclear research reactor is described. The studies of the radiation defects performed with positron annihilation confirmed that divacancies dominate in the irradiated material. Thermal treatment of irradiated silicon at $700-1000^{\circ} \mathrm{C}$ produces void-phosphorus complexes and void aggregates. The resistivity of the samples produced by neutron transmutation doping was found to be uniform within $2.5 \%$ limits. The severe reduction of the minority carrier lifetime in irradiated samples was confirmed.

PACS numbers: 81.40.Wx, 71.60.-i, 78.70.Bj, 61.80.--x

\section{Introduction}

Due to the complexity of solar grade multicrystalline silicon its physical properties are interesting field of research of modern materials engineering [1]. During crystallization process, contaminations aggregate in liquid phase of silicon and precipitate at the borders of grains. Therefore, their presence determines the quality of multicrystalline material. They host the centers of recombination that decrease minority carrier's lifetime $[2,3]$. One of the main problems during silicon crystallization is spatial homogeneity of the dopant distribution, which decides on the type of electrical conductivity and value of specific resistivity of

*corresponding author; e-mail: c.pochrybniak@cyf.gov.pl 
material. Useful level of the dopant concentration in silicon ranges from $10^{15}$ to $10^{16} \mathrm{~cm}^{-3}$ [4]. The differences in specific resistivity in the sample reach several percent, after traditional doping in silicon crystallization process. Metallic contaminations, "lifetime killers" of minority carriers, are in solar grade silicon at the level of $10^{11}-10^{13} \mathrm{~cm}^{-3}$. Since the resistivity of silicon for solar cell should be $0.5-10 \Omega \mathrm{cm}$, the concentration of the dopant atoms must be at $10^{15}$ to $10^{16} \mathrm{~cm}^{-3}$. Gettering the parasitic contaminations and passivation of recombination centers requires additional thermal processes. However, intensive thermal processes of multicrystalline silicon decrease the mobility of minority carriers and reduce their lifetime.

Basic nuclear reaction employed in the neutron transmutation doping (NTD) of silicon consists in transformation of silicon nuclei to phosphorus ones after capture of thermal neutron

$$
{ }_{14}^{30} \mathrm{Si}(\mathrm{n}, \gamma){ }_{14}^{31} \mathrm{Si} \quad \stackrel{2.62 \mathrm{~h}}{\longrightarrow} \quad{ }_{15}^{31} \mathrm{P}+\beta^{-} .
$$

The reaction is used mainly in production of semiconductor power circuits and $\gamma$-ray detectors. The non-uniform spatial distribution of dopant in irradiated sample of $\mathrm{Si}$ is due to the self-shielding effect and is not a problem even for large crystals.

During irradiation the crystal defects are produced in transmutation of silicon. Defects can be removed by thermal annealing processes in the solar cells manufacturing. It is expected that the basic merits of NTD, i.e. spatial homogeneity and small phosphorus concentration between borders of grains in basic material, will improve solar cells parameters.

In Sect. 2 we give a short description of the implementation of NTD technique in MARIA reactor. The samples are described in Sect. 3. The results of positron annihilation studies of the defects introduced by radiation are presented in Sect. 4.1. The distribution of resisitivity across samples is presented in Sect. 4.2.

\section{NTD procedure}

In order to obtain appropriate concentration of phosphorus in reasonable time, the high thermal neutron flux density is needed. The resistivity below $1 \Omega \mathrm{cm}$ can be reached in $100 \mathrm{~h}$ with the thermal neutron flux density of $10^{14} \mathrm{~cm}^{-2} \mathrm{~s}^{-1}$. This flux density is met in the core of the nuclear research reactor MARIA in the vicinity of fuel elements, at first row of the beryllium reflector. The necessary irradiation conditions are provided in $\varnothing 60$ irradiation channel in beryllium block denoted as J-IX in the reactor core matrix.

Before irradiations the thermal neutron flux density and epithermal index were determined. As activation detectors foils of alloys of gold with aluminum and cobalt were used. The determined total neutron flux density was $1.0 \times 10^{14} \mathrm{~cm}^{-2} \mathrm{~s}^{-1} \pm 4.8 \%$, and epithermal index was $0.05 \pm 0.02$. The determined epithermal index indicates that there is appreciable fraction of the fast neutrons in the total neutron flux present. One should expect that the fast neutrons 
might generate high density of radiation defects within the bulk of the irradiated material.

Distribution of thermal neutron flux density along the axis of the irradiation channel was determined by the copper wire activation method. The distribution exhibits marked maximum at $660 \mathrm{~mm}$ depth from upper edge of beryllium block. In the $100 \mathrm{~mm}$ region containing that maximum the neutron flux density is almost constant within $2 \%$. The size of the region matches the sample height. The self-shielding effect of silicon estimated from distribution of neutron flux density in the neutron reflector region yields for 2 inch diameter samples the 5 to $8 \%$ radial differences in doping. In order to decrease this effect, the samples should be rotated around vertical axis during the irradiation.

\section{Samples}

Silicon wafers cut from floating-zone method produced single-crystalline and multicrystalline materials of 500-800 $\Omega \mathrm{cm}$ resistivity were used. The wafers were of 2 inch diameter and $0.5 \mathrm{~mm}$ thick disks. Two series differing in irradiation time were carried out. The thermal neutron dose for the irradiated samples was determined with special activation detectors (foil $\mathrm{AlCo}$ ) placed between samples. The expected resistivity after irradiation of the silicon was estimated from the determined neutron dose by standard procedure [5].

For the first set of samples, irradiated for $100 \mathrm{~h}$, the values of thermal neutron dose and expected resistivity were

activation detector No. $13.4 \times 10^{19} \mathrm{~cm}^{-2} \rho=0.77 \Omega \mathrm{cm}$,

activation detector No. $23.7 \times 10^{19} \mathrm{~cm}^{-2} \rho=0.72 \Omega \mathrm{cm}$,

determined with the error of $5.2 \%$ in both cases.

For the second set of samples, irradiated for $42 \mathrm{~h}$ the neutron dose and expected resistivity were

activation detector No. $3 \quad 1.31 \times 10^{19} \mathrm{~cm}^{-2} \quad \rho=2 \Omega \mathrm{cm}$,

and the error of measurement was $2.5 \%$.

The silicon samples were "cooled" during the week after irradiation. Only slim contaminations from the second circulation of reactor cooling water were noticed, i.e. ${ }^{46} \mathrm{Sc},{ }^{51} \mathrm{Cr},{ }^{103} \mathrm{Ru}$, and ${ }^{141} \mathrm{Ce}$. After standard cleaning the samples in special acid bath (mixture of citrates and citric acid) and ultrasonic cleaning in water bath, most of contaminations (more than 95\%) were removed.

\section{Results}

The radiation defects introduced into the samples by the nuclear radiation were studied with the positron annihilation. The main part of studies dealt with the single-crystalline samples, because it is not reasonable to compare the defects in different samples of the multicrystalline silicon. The single-crystalline samples 
provide appropriate uniformity of initial material needed for comparison of the radiation defects. It is assumed that the radiation defect density is the same in single- and multicrystalline samples. For comparison the not irradiated silicon samples with resistivity about $1 \mathrm{k} \Omega \mathrm{cm}$ (undoped) and $5 \Omega \mathrm{cm}$ (doped during crystallization) and irradiated samples were investigated.

\subsection{Radiation deffects}

Positron lifetime measurements were made at room temperature with the conventional fast-fast spectrometer of the time resolution of 270 ps for Co-60. The positron source of activity about $740 \mathrm{kBq}$, covered by $5 \mu \mathrm{m} \mathrm{Ni}$ foil, was placed between two pieces of the investigated sample. 10 to 20 lifetime spectra were recorded for each sample. Then the spectra were added using the special procedure which accounted for the drift of the zero channel. With this procedure the very high counts $\left(30-60 \times 10^{6}\right)$ were achieved.

The experimental data were analyzed with the LT-9 program [6] that unlike other codes used in the field, is suitable for fitting not only a single spectrum but also enables simultaneous fits to series of spectra. The simultaneous fitting of many spectra reduces the number of the free parameters if some of them are common for all analyzed spectra.

In our case the series composed of nine spectra each were collected for samples after different annealing procedures. The results were analyzed within the three-state trapping model $[7,8]$. The model takes into account the processes of positron annihilation in the bulk material and at the two types of defects. Within the model the positron lifetime spectrum $S(t)$ consists of three components

$$
S(t)=\sum_{j=0}^{2} I_{j} \lambda_{j} \exp \left(-\lambda_{j} t\right) .
$$

Parameters $\lambda_{0}=1 / \tau_{\mathrm{b}}+\mu_{1} c_{1}+\mu_{2} c_{2}$ and $\lambda_{j}=1 / \tau_{j}(j=1,2)$ denote the positron annihilation rates at bulk and different types of defects (Fig. 1), whereas $I_{j}$ are intensities given by

$$
I_{0}=1-I_{1}-I_{2} \quad \text { and } \quad I_{j}=\frac{\mu_{j} c_{j}}{\lambda_{0}-\lambda_{j}}, \quad j=1,2 .
$$

We assumed that the structure of the bulk material and the two types of defects does not change during different annealing procedures. Therefore, the positron lifetimes $\tau_{\mathrm{b}}, \tau_{1}$, and $\tau_{2}$ do not change with the temperature and duration of annealing. The $\tau_{\mathrm{b}}, \tau_{1}$, and $\tau_{2}$ were determined from fitting the formula (1) to all the spectra of investigated samples. Because annealing changes the defects' concentrations, the fit parameters $\mu_{1} c_{1}$ and $\mu_{2} c_{2}$ were assumed to be different for each sample.

It turned out that in order to obtain a good fit to the data, a long-lived component (about $2 \mathrm{~ns}$ ) with the intensity lower than $1 \%$ had to be incorporated into the description of each spectrum. Moreover, a source correction $(4.5 \%$ of $105 \mathrm{ps}$ and $0.5 \%$ of $320 \mathrm{ps}$ ) was made. 


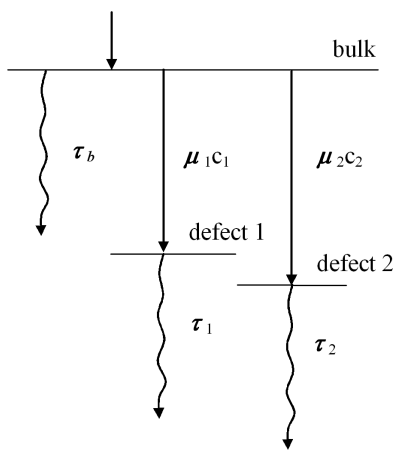

Fig. 1. The scheme of the positron annihilation processes according to the 3 -state trapping model. The horizontal lines represent the free states of positron, i.e. the delocalized state of positron in the bulk of the material and its two localized states in two types of defects. $\tau_{\mathrm{b}}$ is the positron lifetime in bulk material, $\tau_{1}$ and $\tau_{2}$ are the positron lifetimes in two different types of defects, respectively. $\mu_{1} c_{1}$ and $\mu_{2} c_{2}$ are the trapping rates into these defects, which are proportional to their concentrations $c_{1}$ and $c_{2}$.

The determined values of $\tau_{\mathrm{b}}, \tau_{1}, \tau_{2}$ and the long lifetime $\tau_{3}$ obtained from the fitting are shown in Table I. The $\tau_{\mathrm{b}}$ is lower by about $25 \mathrm{ps}$ from the most frequently observed value of $218-220$ ps for pure silicon [8-11]. Nevertheless, it is in the range of theoretical values (186 and $221 \mathrm{ps}$ ) predicted by different theoretical approaches [8].

TABLE I

The lifetimes of positron in the bulk $\left(\tau_{\mathrm{b}}\right)$ and in two different defect states $\left(\tau_{1}\right.$ and $\left.\tau_{2}\right)$ as well as the lifetime of the long-lived component $\left(\tau_{3}\right)$.

\begin{tabular}{|c|c|c|c|c|}
\hline $\begin{array}{c}\text { Sample } \\
\text { (annealing }[\mathrm{min}] \text {, } \\
\text { temperature }\left[{ }^{\circ} \mathrm{C}\right] \text { ) }\end{array}$ & $\begin{array}{c}\tau_{\mathrm{b}} \\
{[\mathrm{ps}]}\end{array}$ & $\begin{array}{c}\tau_{1} \\
{[\mathrm{ps}]}\end{array}$ & $\begin{array}{c}\tau_{2} \\
{[\mathrm{ps}]}\end{array}$ & $\begin{array}{c}\tau_{3} \\
{[\mathrm{~ns}]}\end{array}$ \\
\hline$\left(30^{\prime}, 500\right),\left(30^{\prime}, 600\right)$ & \multirow{5}{*}{193} & \multirow{5}{*}{295} & \multirow{5}{*}{266} & 1.52 \\
\hline$\left(10^{\prime}, 700\right),\left(30^{\prime}, 700\right)$ & & & & \multirow{4}{*}{2.25} \\
\hline$\left(10^{\prime}, 800\right),\left(30^{\prime}, 800\right)$ & & & & \\
\hline$\left(10^{\prime}, 900\right),\left(30^{\prime}, 900\right)$ & & & & \\
\hline$(30 ', 1000)$ & & & & \\
\hline
\end{tabular}

The lifetime spectra of samples annealed for $30 \mathrm{~min}$ at $500^{\circ} \mathrm{C}$ and $600^{\circ} \mathrm{C}$ contain, in addition to a short-lived component originating from the positron annihilation in bulk, an additional one with the lifetime $\left(\tau_{1}\right)$ of $295 \mathrm{ps}$. Its relative intensity is $68 \%$ and $47 \%$ for 500 and $600^{\circ} \mathrm{C}$, respectively. Since the lifetime pa- 
rameter of this component is exactly the same as the one obtained for neutral divacancies in silicon at $300 \mathrm{~K}[12,13]$ and the reported values of the positron lifetime for those divacancies in Si are contained in the range of 295-330 ps [8-13], it is evident that the component is due to the positron annihilation at neutral divacancies $\left(\mathrm{V}_{2}^{0}\right)$.

For the samples annealed at $700^{\circ} \mathrm{C}$ intensity of the component associated with $\mathrm{V}_{2}^{0}$ decreases to $4.6 \%$ and $3.7 \%$ after 10 and 30 min of annealing, respectively. Additionally, in the lifetime spectra of these samples a new component of lifetime $\tau_{2}=266$ ps was found. We should mention that a component of similar lifetime of about 250 ps was found in NTD silicon samples $[9,11]$ and was ascribed to positron annihilation at E-type defects. The E-type defect is a complex $(\mathrm{V}-\mathrm{P})$ of a monovacancy and an atom of phosphorus. The theoretically calculated value of the positron lifetime in this type of defect is 270 ps [14] that is very close to our experimental result.

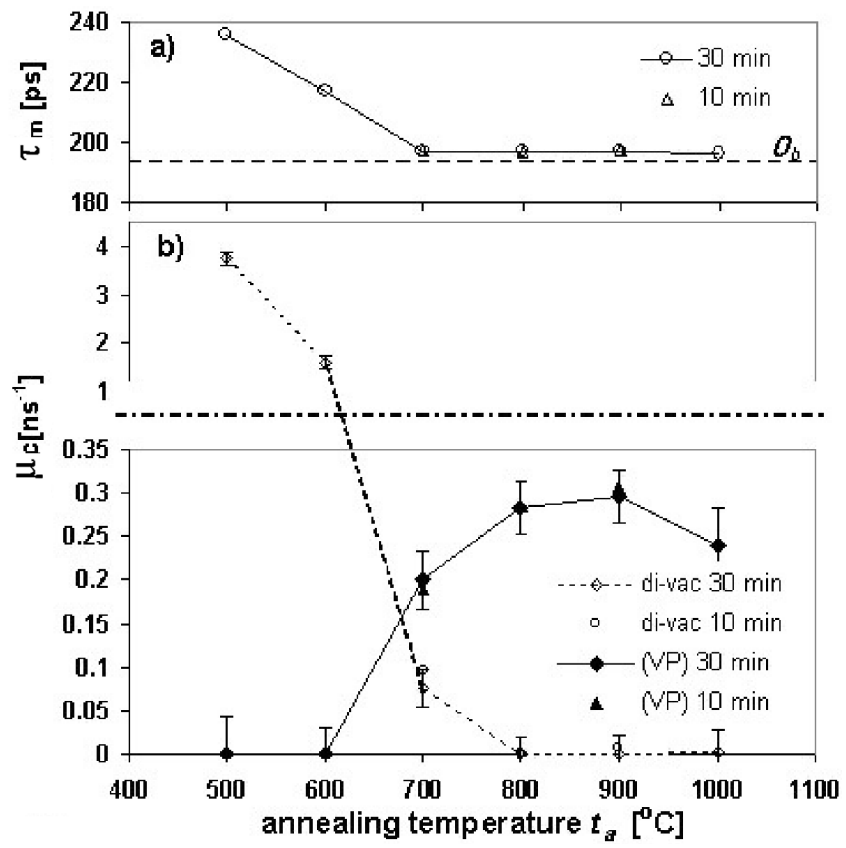

Fig. 2. (a) Mean positron lifetime $\tau_{\mathrm{m}}$ in the investigated samples as a function of the annealing temperature $\left(t_{\mathrm{a}}\right)$ and annealing time (the error bars are hidden in the graphic symbols). The horizontal dashed line represents value of the bulk lifetime $\tau_{\mathrm{b}}$. (b) Dependence of the trapping rates into divacancies (open diamonds and circles) and into $\mathrm{V}-\mathrm{P}$ complexes (solid circles and triangles) on the annealing temperature and time. Because the trapping rate into divacancies below $t_{\mathrm{m}}=700^{\circ} \mathrm{C}$ is much higher than that above this temperature, the graph is divided in two different scales. 
For samples annealed at $800^{\circ} \mathrm{C}$ and $900^{\circ} \mathrm{C}$ the contribution of $\mathrm{V}_{2}^{0}$ component disappears and the component due to annihilation at V-P complexes stabilizes at $17 \%$, for any annealing time studied. For samples annealed at $1000^{\circ} \mathrm{C}$ part of E-type defects is removed yielding the decrease in the corresponding spectrum component to $14.5 \%$.

The global changes in the positron lifetime spectrum are revealed in the behavior of the mean positron lifetime $\tau_{\mathrm{m}}$ that is determined with high precision independently of the model used. We found that for our samples $\tau_{\mathrm{m}}$ decreases drastically for annealing temperatures $t_{\mathrm{m}}>500^{\circ} \mathrm{C}$ (Fig. 2a). The decrease can be easily explained as annealing of $\mathrm{V}_{2}$ defects. Since the asymptotic value of $\tau_{\mathrm{m}}$ is higher than $\tau_{\mathrm{b}}$, another type of defects should be present in samples annealed above $600^{\circ} \mathrm{C}$. The evolution of the defect concentration in the investigated samples is described by the changes of the positron trapping rates $\mu_{1} c_{1}$ and $\mu_{2} c_{2}$ into $V_{2}^{0}$ and $\mathrm{V}-\mathrm{P}$ complexes, respectively (Fig. $2 \mathrm{~b}$ ). The concentration of $\mathrm{V}_{2}$ decreases sharply if the annealing temperature $t_{\mathrm{m}}$ exceeds $500^{\circ} \mathrm{C}$. The $\mathrm{V}_{2}^{0}$ defects practically disappear for $t_{\mathrm{m}}>700^{\circ} \mathrm{C}$. On the other hand, the V-P complexes are not observed for $t_{\mathrm{m}}$ below $600^{\circ} \mathrm{C}$. They start to form in samples annealed at $t_{\mathrm{m}}>600^{\circ} \mathrm{C}$. Their concentration increases when the annealing temperature is increased up to $t_{\mathrm{m}} \approx 900^{\circ} \mathrm{C}$ and above this temperature it decreases gradually.

On the basis of the determined positron trapping rates the concentrations of discussed defects were estimated (Table II) using the positron trapping constants for $\mathrm{V}_{2}^{0}\left(\mu_{1}=1.5 \times 10^{-8} \mathrm{~cm}^{3} \mathrm{~s}^{-1}[15]\right)$ and $(\mathrm{V}-\mathrm{P})^{0}\left(\mu_{2}=1.4 \times 10^{-8} \mathrm{~cm}^{3} \mathrm{~s}^{-1}[16]\right)$.

TABLE II

Estimated values of $\mathrm{V}_{2}^{0}$ and $\mathrm{V}-\mathrm{P}$ concentration after anealing of samples for 10 and $30 \mathrm{~min}$ at different temperatures.

\begin{tabular}{c|c|c|c}
\hline \hline $\begin{array}{c}\text { Annealing time } \\
{[\mathrm{min}]}\end{array}$ & $\begin{array}{c}\text { Annealing } \\
\text { temperature }\left[{ }^{\circ} \mathrm{C}\right]\end{array}$ & $\begin{array}{c}\text { Concentration } \\
\mathrm{V}_{2}^{0}\left(c_{1}\right)\left[\mathrm{cm}^{-3}\right]\end{array}$ & $\begin{array}{c}\text { Concentration } \\
\mathrm{V}-\mathrm{P}\left(c_{2}\right)\left[\mathrm{cm}^{-3}\right]\end{array}$ \\
\hline 30 & 500 & $2.5 \times 10^{17}$ & $>10^{13}$ \\
30 & 600 & $1.1 \times 10^{17}$ & $>10^{13}$ \\
10 & 700 & $6.4 \times 10^{15}$ & $1.4 \times 10^{16}$ \\
30 & 700 & $5.0 \times 10^{15}$ & $1.4 \times 10^{16}$ \\
10 & 800 & $4.5 \times 10^{13}$ & $2.0 \times 10^{16}$ \\
30 & 800 & $>10^{13}$ & $2.0 \times 10^{16}$ \\
10 & 900 & $>10^{13}$ & $2.2 \times 10^{16}$ \\
30 & 900 & $>10^{13}$ & $2.1 \times 10^{16}$ \\
30 & 1000 & $>10^{13}$ & $1.7 \times 10^{16}$
\end{tabular}

The contribution of the longest-lived component (with lifetime $\tau_{3}=1.52 \mathrm{~ns}$ ) in the annihilation spectra for samples annealed at $500^{\circ} \mathrm{C}$ and $600^{\circ} \mathrm{C}$ is equal 
to $0.78 \%$. This component decreases to $0.41 \%$ for the samples annealed in $700^{\circ} \mathrm{C}$ and $1000^{\circ} \mathrm{C}$ with simultaneous increase in $\tau_{3}$ to 2.25 ns. Similar long-lived components were observed previously [11]. We suppose that such components arise from positronium annihilation in small voids created by the aggregations of vacancies. It is possible to relate the lifetime of positronium with the size of the void positronium annihilates in using an empirical formula given in [17]. According to that $\tau_{3}=1.52 \mathrm{~ns}$ corresponds to the void radius $0.24 \mathrm{~nm}$, and $\tau_{3}=0.25 \mathrm{~ns}$ corresponds to the void radius $0.31 \mathrm{~nm}$.

We conclude that annealing of silicon at $700-1000^{\circ} \mathrm{C}$ leads to generation of $\mathrm{V}-\mathrm{P}$ complexes and probably to clustering of void-type defects into big size vacancies aggregations mainly at the expense of the population of divacancies, which are dominant type of defects in non-annealed samples of neutron irradiated silicon.

\subsection{Resistivity}

The resistivity measurements were performed on the irradiated samples annealed at $500-1000^{\circ} \mathrm{C}$ for $10-30 \mathrm{~min}$ in rapid thermal annealing furnace. Resistivity was measured by four-needle probe. The resistivity of the annealed material strongly depends on temperature and duration of the process (Fig. 3). We found that the best conditions for radiation damage annealing was $800^{\circ} \mathrm{C}$ and $20 \mathrm{~min}$. The resistivity of the annealed NTD samples were $\approx 1 \Omega \mathrm{cm}$ for the samples produced in the $100 \mathrm{~h}$ irradiation run and $\approx 3 \Omega \mathrm{cm}$ for the samples irradiated for $42 \mathrm{~h}$. It is evident that the determined resistivities matched the values estimated in Sect. 3 on the basis of the neutron dose [5].
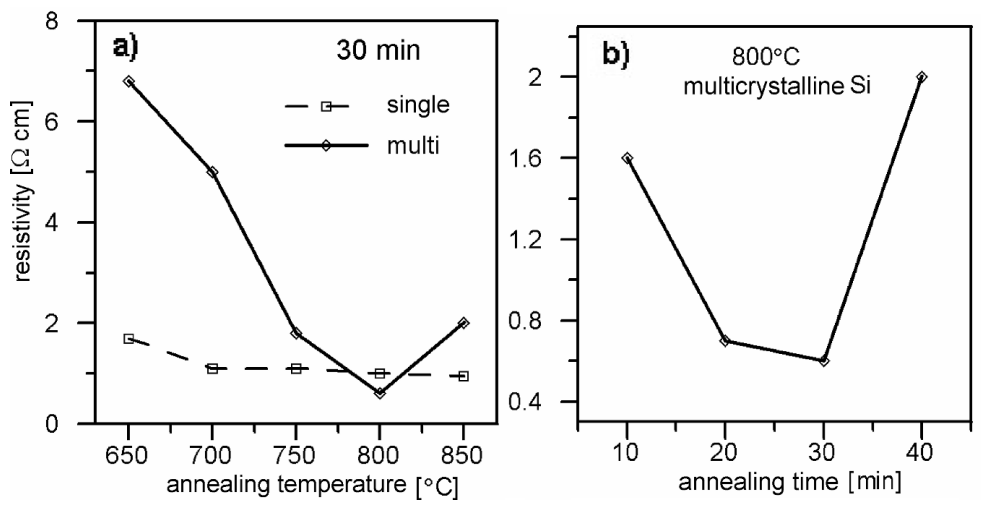

Fig. 3. Dependence of the final resistivity of silicon samples on the temperature (a) and time of annealing (b).

The uniformity of the resistivity distribution in sample annealed at $800^{\circ} \mathrm{C}$ for 20 min was studied with the bulk photovoltaic effect (BPV) produced by the gradients of resistivity [18]. For comparison, the sample doped during crystallization was studied. The measurements were performed on the $50 \mathrm{~mm}$ diameter 
samples of $\approx 3 \Omega \mathrm{cm}$ resistivity. The light probe was of $0.2 \mathrm{~mm}$ diameter. The amplitude of the fluctuations in the profiles of the BPV voltage are for NTD samples half of those observed in the samples doped during crystallization (Fig. 4). The resistivity variations across sample calculated on the basis of the BPV data for the NTD sample are confined within $2.5 \%$ error band (Fig. 5).

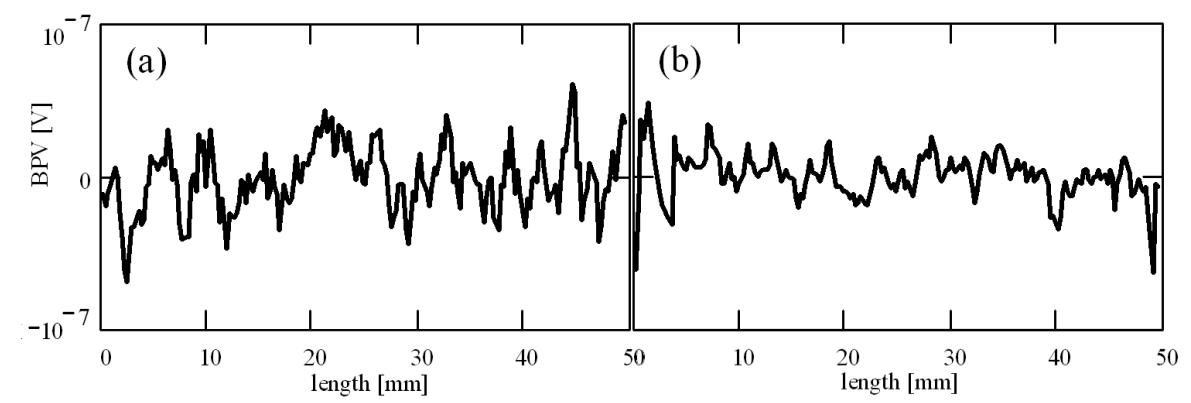

Fig. 4. Profiles of the photoelectric voltage (BPV) along the diameter of the Si wafer measured for the sample doped during crystallization (a) and the sample doped by the neutron transmutation (b).

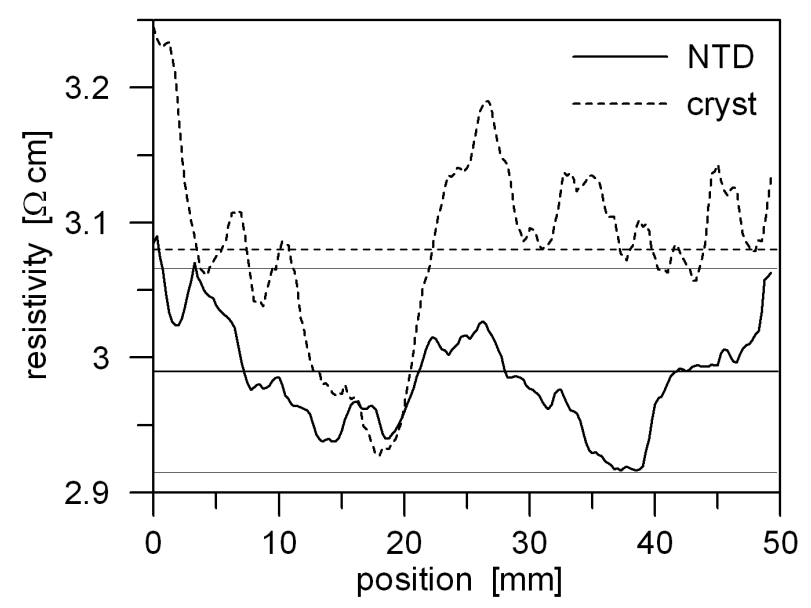

Fig. 5. The spatial distribution of the resistivity along sample diameter determined from BPV data for NTD sample (solid line) and the sample doped during crystallization (dotted line). The horizontal lines denote the average resistivity of each sample. The thin solid lines are $\pm 2.5 \%$ error band limits for NTD sample.

\subsection{Minority lifetime carrier}

Preliminary measurements of the minority carriers' lifetime have been performed with photoconductivity decay technique for the samples studied. The 
measurements revealed substantial decrease in minority carrier lifetime to $\approx 1 \mu \mathrm{s}$ in the NTD samples. It attains only approximately $1 \%$ of the minority carrier lifetime value obtained for the material before irradiation. This effect is attributed to high density of radiation defects $[19,20]$. It is the main problem in application of neutron doped material in solar cell devices. We hope that the effect can be reduced with application of softer neutrons, i.e. neutrons of lower epithermal index, in irradiation. This can be accomplished by performing irradiation outside the reactor core in the reflector region.

\section{Conclusions}

Our resistivity studies proved that the nuclear transmutation doping technique provides good uniformity of the irradiated samples. It was confirmed that divacancies are dominant type of defects in the neutron irradiated silicon. Annealing of the samples at $700-1000^{\circ} \mathrm{C}$ produces V-P complexes generation and void-type defects clustering into big size vacancies aggregations. Since the strong reduction of minority carriers' lifetime is the main problem of the application of NTD material in solar cells, the effect should be studied for different irradiation conditions.

\section{Acknowledgments}

This work was partially financed under the Polish Ministry of Science and Higher Education research grants N508 048 31/2569 and N515 088433.

\section{References}

[1] L.J. Geerlings, P. Manshanden, G.P. Wyers, E.J. Øvrelid, O.S. Raaness, A.N. Waernes, B. Wiersma, in: Proc. 20th European PV Solar Energy Conf. and Exhibition, Barcelona (Spain) 2005, Eds. W. Paltz, H. Ossenbrink, P. Helm, Barcelona 2005, p. 619.

[2] D. Sarti, R. Einhaus, Solar Energy Mater. Solar Cells 72, 27 (2002).

[3] A. Cuevas, Mater. Forum 27, 1 (2004).

[4] W.R. Runyan, Silicon Semiconductor Technology, McGraw-Hill Book Company, New York 1965.

[5] N.W. Crick, H. Blowfield, in: Silicon Transmutation Doping Techniques and Practice, IAEA-TECDOC-456, IAEA, Vienna 1988, p. 65.

[6] J. Kansy, Nucl. Instrum. Methods Phys. Res. A 374, 235 (1996).

[7] W. Brandt, R. Paulin, Phys. Rev. B 5, 2430 (1972).

[8] R. Krause-Rehberg, H.S. Leipner, Positron Annihilation in Semiconductors, Defect Studies, Springer Series in Solid State Science, Springer, Berlin 1999.

[9] X.T. Meng, A.K. Liolios, M. Chardalas, Sp. Dedoussis, C.A. Eleftheriadis, Stef. Charalambous, Phys. Lett. A 157, 73 (1991).

[10] W. Puff, X.T. Meng, J. Appl. Phys. 73, 648 (1993). 
[11] M. Coeck, N. Balcaen, T. Van Hoecke, B. Van Waeyenberge, D. Segers, C. Dauwe, C. Laermans, J. Appl. Phys. 87, 3674 (2000).

[12] M. Hasegawa, T. Chiba, A. Kawasuso, T. Akahane, M. Suezawa, S. Yamaguchi, K. Sumino, Mater. Sci. Forum 196-201, 1481 (1995).

[13] A. Kawasuso, M. Hasegawa, M. Suezawa, S. Yamaguchi, K. Sumino, Hyperfine Interact. 84, 397 (1994).

[14] A. Polity, F. Börner, S. Huth, S. Eichler, R. Krause-Rehberg, Phys. Rev. B 58, 10363 (1998).

[15] R. Krause-Rehberg, G. Dlubek, A. Polity, Mater. Sci. Forum 196-201, 1649 (1995).

[16] A. Kawasuso, M. Hasegawa, M. Suezawa, S. Yamaguchi, K. Sumino, Jpn. J. Appl. Phys. Pt. 1 34, 2197 (1995).

[17] M. Eldrup, D. Lightbody, J.N. Sherwood, Chem. Phys. 63, 51 (1981).

[18] S. Sikorski, T. Piotrowski, Progr. Quant. Electron. 27, 295 (2003).

[19] G.C. Messenger, M.S. Ash, The Effects of Radiation on Electronic Systems, Van Nostrand Reinhold, New York 1992.

[20] G.C. Messenger, IEEE Trans. Nucl. Sci. 39, 468 (1992). 